\title{
Preparation of Graphene Oxide from Lignin by Gel Combustion Method and Its Performance as Supercapacitor
}

\author{
Rajapandian Rajagopal ${ }^{1}$, Masaharu Komiyama ${ }^{1,2,3, *}$ and Azry Borhan $^{1,2}$ \\ ${ }^{1}$ Chemical Engineering Department, Universiti Teknologi PETRONAS, 32610 Seri Iskandar, Perak Darul Ridzuan, Malaysia \\ ${ }^{2} \mathrm{HICoE}-$ Centre for Biofuel and Biochemical Research, Institute for Sustainable Building, Universiti Teknologi PETRONAS, 32610 Seri \\ Iskandar, Perak Darul Ridzuan, Malaysia \\ ${ }^{3}$ Clean Energy Research Center, University of Yamanashi, Takeda, Kofu, 400-8511, Japan
}

\begin{abstract}
Carbon materials derived from biomass are drawing increasing attention due to its advantages in economical as well as ecological production. Among the biomass resources to be utilized for that purpose, lignin possesses unique characteristics: it is available in bulk quantity as a low-value by-product from the pulp and paper industries and its chemical structure based on complex aromatic networks provides excellent precursor for grahitic carbon materials such as graphene. The existing techniques to convert lignin to carbon materials require long processing time, complex steps and higher temperatures, thus increasing the production cost and hindering its commercialization. Here an alternate low-cost, one-pot synthesis method utilizing organic solvent gel combustion technique is developed to yield crystalline graphene oxide as an end product. Capacitor performance of the developed graphene oxide was investigated in terms of the parameters involved in the synthesis procedure.
\end{abstract}

\section{Introduction}

Demand for environmental friendliness and renewable nature on energy sources have been increasingly prominent in recent years with the heightening concerns about climate neutrality as well as depletion of natural resources [1-5]. Supercapacitors, a type of electrochemical capacitors, have attracted much attention as one of the promising energy storage system for leveling renewable power fluctuations, because of their outstanding properties such as excellent cycle performance, fast charging/discharging capability and high power density [6-9]. The performance of supercapacitors strongly depends on the electrode employed, and carbon materials including graphene, activated carbon, carbon nanotube and so on are among the candidates sought after for the purpose [10-12].

A potential renewable source for the carbon materials is lignocellulosic biomass [13]. In Malaysia, for instance, about 5.4 million hectare of the oil palm agricultural land generates about $80 \%$ of the biomass, making it one of the largest contributors of lignocellulosic biomass in the world [14]. On the other hand, crude lignin is obtained in large quantities in the pulp and paper industries, mainly as Kraft lignin and lignosulfonate. Kraft lignin is the largest raw lignin byproduct produced from the pulping process. As a low value compound, it has so far mainly been used as a fuel source in combustion processes and less than $5 \%$ of its world supply has been used for other purposes $[15,16]$. Therefore, valorization of lignin into value-added chemicals or materials suitable for large- scale industrial applications has been the focus of many researches [17].

Lignin is regarded as a starting core for synthesis of functional carbon materials, and numbers of functional carbon materials like carbon fibers and porous carbon with different functionalities can be synthesized from it through thermochemical methods such as pyrolysis or hydrothermal carbonization. Synthesis of amorphous (activated carbon and carbon black) and crystalline (carbon nanotubes/nanofibers and graphene) carbon materials with controllable functionality often involve tedious synthetic process. Furthermore, most of these methods require fossil fuel-based chemical precursors (e.g., $\mathrm{CH}_{4}, \mathrm{C}_{2} \mathrm{H}_{2}$, and $\mathrm{C}_{2} \mathrm{H}_{4}$ ), metal catalysts, and/or complicated apparatus involving high processing temperatures, which are not necessarily environmentally friendly or economically sustainable [16].

Among those carbon materials, graphene has carbon atoms in honeycomb structure and shows interesting characteristics with excellent electrical and mechanical properties $[18,19]$. It attracts significant attention due to their potential applications in conductors, batteries, catalysts and biosensors. Common production method for graphene is by mechanical exfoliation, epitaxial growth and chemical vapor deposition [20]. The largescale production of graphene from lignin available globally, however, is bottlenecked due to the high cost of its production.

In search of a low-cost process for graphene synthesis from lignin, gel combustion method is chosen in the current study. The citric acid sol-gel combustion method is one of non-alkoxide sol-gel methods, which is 
efficient for the preparation of nanocrystalline inorganic powders. The attractive features of this method are its ability to synthesize materials with high purity, better homogeneity and high surface area in a single step [21]. Even though the concept of gel autocombustion is universal, the methods to be applied may not be the same for all the materials. Thus in this research, synthesis procedure utilizing gel combustion method will be developed to obtain crystalline carbon material graphene oxide as an end product from Kraft lignin, and their supercapacitance performance will be evaluated based on the synthesis parameters.

\section{Experimental Procedure}

Lignin purchased from Tokyo Chemical Industry was used as a starting carbon material and A.R. grade citric acid, ethylene glycol, magnesium nitrate purchased from Sigma Aldrich were used as chelating, gelation and combustion agent, respectively. Potassium hydroxide was selected as solvent to dissolve the raw material and other chemical agents.

Lignin conversion procedure for graphene adopted here is as follows.

- Kraft lignin was kept in an oven at $105^{\circ} \mathrm{C}$ for $2 \mathrm{~h}$ to remove its moisture.

- Calculated amount of Kraft lignin and magnesium nitrate were added, followed by citric acid, into the solvent and stirred for $1 \mathrm{~h}$ with magnetic stirrer to prepare clear solution.

- Ethelene glycol was added into the solution at 1:1 volume ratio.

- The prepared composition was placed on a hot plate at $80{ }^{\circ} \mathrm{C}$ and kept stirring to maintain the homogeneity of the mixture.

- Depending on the volume of the initial solution, the gelation time differs.

- Continued the heating even after gelation which will removes the moisture and powder remained at the bottom.

- Once moisture was removed autoignition started and whole gel will be combusted and turn into carbon powder.

- Synthesized samples were washed with hydroxide solution to eliminate magnesium.

- Prepared samples were washed with acetone several times.

- When necessary, potassium hydroxide washing was also performed.

Prepared graphene samples were examined by Raman spectroscopy (Horiba Jobin Yvon HR800), and their capacitance by cyclic voltammetry (PARSTAT 4000, Princeton Applied Research, India.).

\section{Results and Discussion}

\subsection{Appearance of Starting Lignin and Produced Graphene}

Figures 1 and 2 show physical appearances of the starting lignin material and the synthesized graphene samples, one as synthesized and the other $\mathrm{KOH}$-washed.

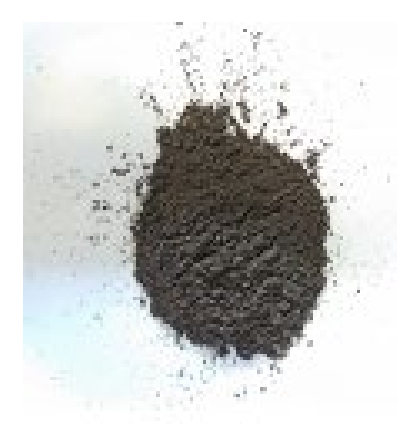

Fig. 1. Starting Kraft lignin.
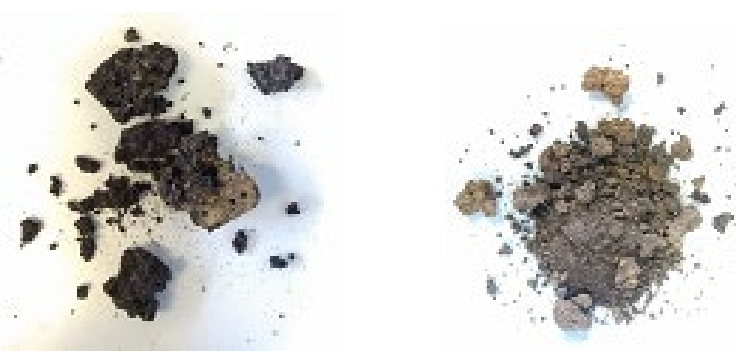

Fig. 2. Synthesized graphene samples (left: as prepared, right: $\mathrm{KOH}$-washed).

\subsection{Raman Spectroscopy}

Figures 3 and 4 exemplify Raman spectra of the produced graphene shown in Fig. 2 using laser excitation at $2.41 \mathrm{eV}$. Prominent features in the spectra are the socalled $\mathrm{G}$ band of monolayer graphene appearing at about $1590 \mathrm{~cm}^{-1}$ and the $2 \mathrm{D}$ band at about $2700 \mathrm{~cm}^{-1}$. We also see the so-called disorder-induced $\mathrm{D}$ band at about half of the frequency of the 2D band (around $1360 \mathrm{~cm}^{-1}$ ) for the samples disordered or with fair amount of graphene edges.

Referring to Figs. 3 and 4, the above observation in Raman spectra of the prepared sample lies in the same peak ranges of the reported graphene Raman shift [22]. It ensures that the synthesized samples contain graphene family, evidencing that the prepared samples are fully converted into graphene material. 


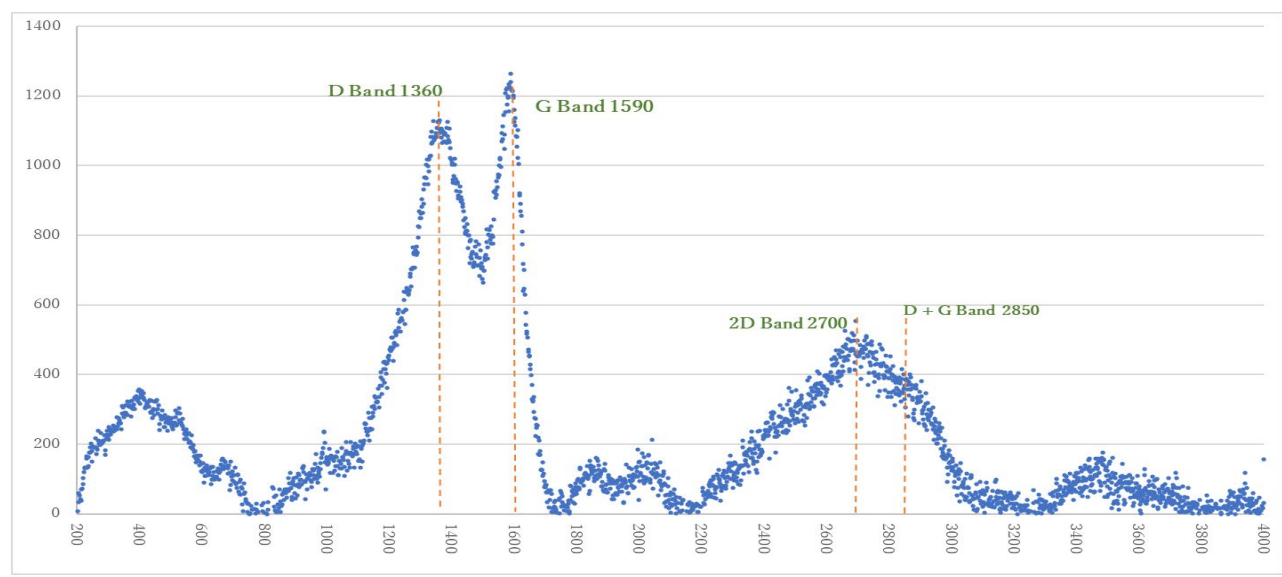

Fig. 3. Raman Spectrum for the as synthesized sample.

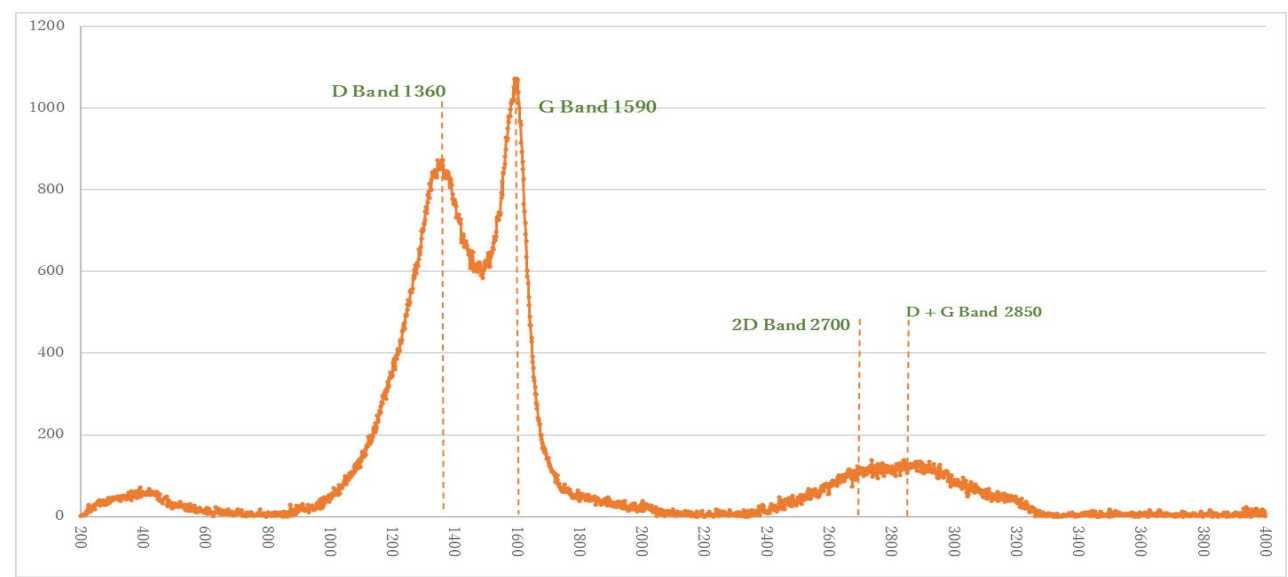

Fig. 4. Raman Spectrum for $\mathrm{KOH}$ washed synthesized sample.

\subsection{Cyclic Voltammetry}

Figures 5 and 6 show cyclic voltammograms of the two samples shown in Fig. 2 and their Raman spectra indicated in Figs. 3 and 4. On the left of each figure cyclic voltammograms of the synthesized graphene materials are shown with different sweeping rates, and on the right, capacitance measurement with different amperages. Table 1 summarizes the capacitance data for these two samples at various current.
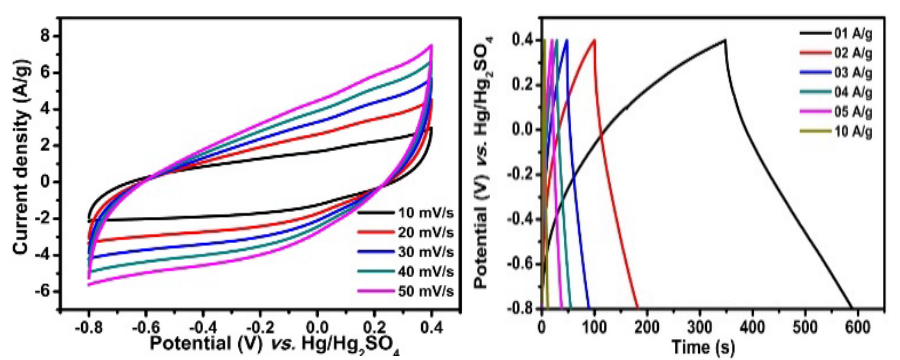

Fig. 5. Left: Cyclic voltammogram of as-synthesized sample under different scan rates. Right: Specific capacitance at 1-10 $\mathrm{A} / \mathrm{g}$.
Capacitance data found in Table 1 indicate that washing of as-synthesized sample by $\mathrm{KOH}$ for $\mathrm{Mg}$ removal decreases its performance compared to the asprepared sample. It is due to the contribution of $\mathrm{Mg}$ metal traces that as-prepared sample exhibits higher performance compared to $\mathrm{Mg}$-removed sample, although the latter should contain potassium ion traces in the sample.
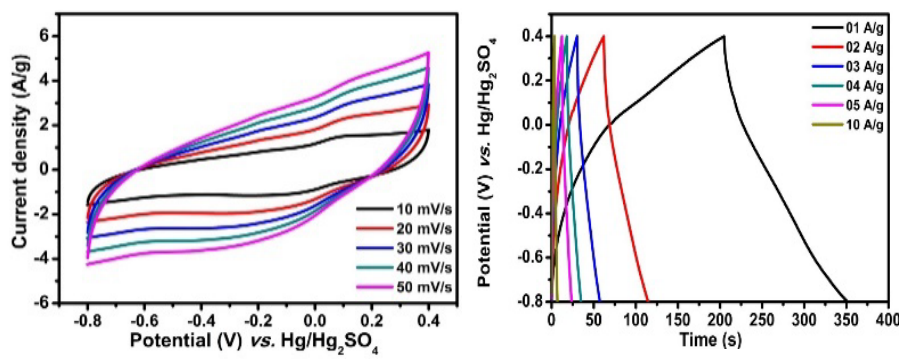

Fig. 6. Left: Cyclic voltammogram of $\mathrm{KOH}-$ washed synthesized samples under different scan rates. Right: Specific capacitance at 1-10 A/g. 
Table 1. Capacitance performance of the two samples.

\begin{tabular}{ccccccc}
\hline \multirow{2}{*}{ Sample } & \multicolumn{6}{c}{ Specific capacitance at following current } \\
& \multicolumn{7}{c}{ densities (A/g) } \\
\cline { 2 - 7 } & 1 & 2 & 3 & 4 & 5 & 10 \\
\hline As synthesized & 192 & 138 & 102 & 90 & 75 & 60 \\
\hline KOH washed & 117 & 87 & 65 & 60 & 46 & 33 \\
\hline
\end{tabular}

\section{Conclusions}

The present research indicated that lignin can be converted into graphene material in one-pot synthesis using gel combustion method. The prepared samples showed promising performance for the application in the field of supercapacitance.

Further exploration on the graphene oxide doping with various metal dopants will enhance its supercapacitance performance. Those efforts will be helpful in commercializing the lignin biomass conversion process, which will expand the valorization of lignin biomass.

\section{Acknowledgement}

Support from the Ministry of Education Malaysia through HICoE award to Centre for Biofuel and Biochemical Research is duly acknowledged.

\section{References}

[1] Kurnia, J.C.; Jangam, S.V.; Akhtar, S.; Sasmito, A.P.; Mujumdar, A.S. Advances in biofuel production from oil palm and palm oil processing waste: A review. Biofuel Res. J. 9, 332-346 (2016).

[2] Asadieraghi, M.; Daud, W.M.A.W., Characterization of lignocellulosic biomass thermal degradation and physiochemical structure: Effects of demineralization by diverse acid solutions. Energy Convers. Manag. 82, 71-82 (2014).

[3] Doherty, W.O.S.; Mousavioun, P.; Fellows, C.M. Value-adding to cellulosic ethanol: Lignin polymers. Ind. Crops Prod. 33, 259-276 (2011).

[4] Aro, T.; Fatehi, P. Production and application of lignosulfonates and sulfonated lignin. Chem. Sus. Chem., 10, 1861-1877 (2017).

[5] Ragauskas, A.J.; Beckham, G.T.; Biddy, M.J.; Chandra, R.; Chen, F.; Davis, M.F.; Davison, B.H.; Dixon, R.A.; Gilna, P.; Keller, M.; Langan, P.; Naskar, A.K.; Saddler, J.N.; Tschaplinski, T.J.; Tuskan, G.A.; Wyman, C.E. Lignin valorization: Improving lignin processing in the biorefinery. Science, 344, 1246843 (2014).

[6] Thakur, S.; Govender, P.P.; Mamo, M.A.; Tamulevicius, S.; Mishra, Y.K.; Thakur, V.K. Progress in lignin hydrogels and nanocomposites for water purification: Future perspectives. Vacuum, 146, 342-355 (2017).

[7] Schwierz, F. Graphene transistors. Nature Nanotechnol, 5, 487-496 (2010).

[8] Watcharotone, S.; Dikin, D.A.; Stankovich, S.; Piner, R.; Jung, I.; Dommett, G.H.B.; Evmenenko, G.; Wu,
S.-E.; Chen, S.-F.; Liu, C.-P.; SonBinh T. Nguyen, S.T.; Ruoff, R.S. Graphene-silica composite thin films as transparent conductors. Nano Lett., 7, 18881892 (2007).

[9] Wang, H.; Cui, L.-F.; Yang, Y.; Casalongue, H.S.; Robinson, J.T.; Liang, Y.; Cui, Y.; Dai, H, $\mathrm{Mn}_{3} \mathrm{O}_{4}-$ graphene hybrid as a high-capacity anode material for lithium ion batteries. J. Am. Chem. Soc., 132, 13978-13980 (2010).

[10] Liang, Y.; Li, Y.; Wang, H.; Zhou, J.; Wang, J.; Regier, T.; Dai, $\mathrm{H} . \mathrm{Co}_{3} \mathrm{O}_{4}$ nanocrystals on graphene as a synergistic catalyst for oxygen reduction reaction. Nature Mater., 10, 780-786 (2011).

[11] Wu, L.; Chu, H.S.; Koh, W.S.; Li, E.P. Highly sensitive graphene biosensors based on surface plasmon resonance. Opt. Express, 18, 14395-14400 (2010).

[12] Choi, W.; Lahiri, I.; Seelaboyina, R.; Kang, Y.S. Synthesis of graphene and its applications: A review. Crit. Rev. Solid State Mater. Sci., 35, 52-71 (2010).

[13] Gosselink, R.J.A.; de Jong, E.; Guran, B.; Abächerli, A. Co-ordination network for ligninstandardisation, production and applications adapted to market requirements (EUROLIGNIN). Ind. Crops Prod. 20, 121-129 (2004).

[14] Loh, S.K. The potential of the Malaysian oil palm biomass as a renewable energy source. Energy Conv. Manag., 141, 285-298 (2016).

[15] Abdelaziz, O.Y.; Brink, D.P.; Prothmann J.; Ravi K.; Sun M.; García-Hidalgo, J.; Sandahl, M.; Hulteberg, C.P.; Turner, C.; Lidén, G.; GorwaGrauslund, M.F. Biological valorization of low molecular weight lignin, Biotechnol. Adv., 34, 1318-1346 (2016).

[16] Liu, W.-J.; Jiang H.; Yu, H.-Q. Thermochemical conversion of lignin to functional materials: a review and future directions. Green Chem., 17, 4888-4907 (2015).

[17] Li, J.; Yan, Q.; Zhang, X.; Zhang, J.; Cai, Z. Efficient Conversion of lignin waste to high value bio-graphene oxide nanomaterials. Polymers, 11, 623 (2019).

[18] Allen, M.J.; Tung, V.C.; Kaner, R.B. Honeycomb carbon: A review of graphene. Chem. Rev, 110, 132-145 (2009)

[19] Kumar, A.; Anushree, Kumar, J.; Bhaskar, T. Utilization of lignin: A sustainable and eco-friendly approach, J. Energy Inst., 93, 235-271 (2020).

[20] Mehravar, S.; Fatemi, S.; Komiyama, M. The role of cerium intercalation in the efficient dry exfoliation of graphene layers at a low temperature. Diamond Relat. Mater., 101, 107615 (2020).

[21] Chervin, C.N.; Clapsaddle, B.J.; Chiu, H.W.; Gash, A.E.; Satcher, J.H.; Kauzlarich, S.M. A Non-alkoxide sol-gel method for the preparation of homogeneous nanocrystalline powders of $\mathrm{La}_{0.85} \mathrm{Sr}_{0.15} \mathrm{MnO}_{3}$. Chem. Mater., 18, 7, 1928-1937 (2006).

[22] Wu, J-B.; Lin, M.-L.; Cong, X.; Liu, H.-N.; Tan, P.-H., Raman spectroscopy of graphene-based 
